\title{
Performance and Benchmarking of Multisurface UHF RFID Tags for Readability and Reliability
}

\author{
Joshua Bolton, Erick Jones, Raghavendra Kumar Punugu, Ankan Addy, and Samuel Okate
}

Industrial, Manufacturing, and System Engineering Department, University of Texas at Arlington, 420 Woolf Hall, Arlington, TX 76019, USA

Correspondence should be addressed to Joshua Bolton; joshua.bolton@mavs.uta.edu

Received 16 September 2016; Revised 8 February 2017; Accepted 21 February 2017; Published 5 September 2017

Academic Editor: Aldo Minardo

Copyright (C) 2017 Joshua Bolton et al. This is an open access article distributed under the Creative Commons Attribution License, which permits unrestricted use, distribution, and reproduction in any medium, provided the original work is properly cited.

As the price of passive radio frequency identification (RFID) tags continues to decrease, more and more companies are considering item-level tagging. Although the use of RFID is simple, its proper application should be studied to achieve maximum efficiency and utilization in the industry. This paper is intended to demonstrate the test results of various multisurface UHF tags from different manufacturers for their readability under varying conditions such as orientation of tags with respect to reader, distance of tag from the reader, and materials used for embedding tags. These conditions could affect the reliability of RFID systems used for varied applications. In this paper, we implement a Design for Six Sigma Research (DFSS-R) methodology that allows for reliability testing of RFID systems. In this paper, we have showcased our results about the benchmarking of UHF RFID tags and have put forward an important observation about the blind spots observed at different distances and orientations along different surfaces, which is primarily due to the polarity of the antenna chosen.

\section{Introduction}

Over the years, the prices of the RFID tags have been coming down and the performance of the tags have been drastically going up [1]. Automatic identification of objects has been very prominent in many fields mainly because of ease of use and high efficiency. Auto-ID includes bar codes, smart cards, biometrics and voice recognition, magnetic stripes, RFID, and numerous other devices. However, RFID has many benefits, especially over bar codes; RFIDs are non-line-ofsight and reusable, they can withstand harsh environments, and their high encryption prevents counterfeit [2]. All these features have drawn many organizations to opt for RFID. The implementation of radio frequency identification (RFID) technology is rising and reaching new horizons primarily due to the decreasing cost of RFID tags and its application in diverse fields. The value in potential of these tags is becoming more widely known as companies take an interest to evaluating the life of an RFID tag throughout the supply chain. The mandates of Walmart 2003 and DOD 2004 compelled all their vendors to use RFID in their supply chain, which eventually turned out to be a success for further adoption by other agencies and organizations world-wide. The advantage of RFID is that it requires less hardware and has a larger coverage area [3]. As new technologies continue to emerge into the market, coupled with lower priced, more reliable tags, RFID is becoming enabled to move into areas, other than just the supply chain. Now, there is a lot of demand on the tag manufacturers, and few organizations have in-house expertise to deploy their technology. Thus, many organizations are still depending upon the RFID vendors' assessment of their own products, so this creates the possibility of biased results in terms of tag performance swaying the companies in ways that might not actually benefit their businesses. This means that there is a need for unbiased and reliable sources of information about the tag performance. If this need was left unmet, a door could be opened to misleading claims concerning individual tag performance and unreliable sources of information making way to different companies, which could and has resulted in credibility issues with the RFID end-users [4]. The need for a bench mark of RFID tags is long overdue. A bench 
marking of the field would provide companies with a major advantage in the development and synthesis of RFID tags. These tests of tag performances are repeatable in nature under some assumptions which are mentioned in later part of the paper, to help us compare the performance of different tags, which eventually leads to better decision-making in real time scenario for companies. This paper contributes to the testing of RFID tags in different environments and focuses on tag performance with respect to each parameter. This paper is further divided into several sections, Section 2 comprises Literature Review and contributions, Section 3 discusses the methodology used in this paper, Section 4 discusses results obtained from the methodology, and Section 5 describes the Conclusion and Future work.

\section{Literature Review and Contributions}

Several papers in the literature have proposed different methods for performance and benchmarking of UHF RFID tags. The work by [5] considered maximum range and orientation sensitivity but did not take into consideration the medium on which the tag is put. Reference [6] proposed a method where the system can test the UHF RFID tag's performance to support RFID performance benchmarking automatically and efficiently and to help the user in proper selection of tags for their application. Reference [7] proposed a generalized parameterization of the three-dimensional reading range which can be applied to planning and optimization of readertag network. Reference [8] designed a testing protocol to measure performances of RFID devices for blood supply chain. The work by [9] evaluated RFID systems on performance characteristics as the effective capture zone, tag orientation, tag separation, and so on. The work in [10] proposed a benchmarking protocol given by RFID Global Solutions. The work in [11] designed a testing protocol to measure the performance of RFID devices and benchmarking performances of inlays, handheld RFID readers for application in apparel logistics and retail processes. ISO/IEC 180461:2011 defined test methods for performance characteristics of RFID systems for item management and specified the general requirement and test requirements which helps is selection of devices for application, however, in our research, the main motive was to show how to place the tags on a material for better readability and performance and the significant impact the medium the tag is placed on has on performance than selection of the device for application. The work in [12] assessed the technical performance achievable by RFID technology by carrying out an extensive campaign by considering 14 different stealing patterns for antitheft purpose. However, our research can be extended for antitheft purpose as well based on the readability and reliability of tags. The work in [13] proposed a combinatorial methodology for RFID Benchmarking by taking into consideration distance, vertical and horizontal distance. In this paper, we have devised a standard testing protocol for performance and benchmarking for multisurface UHF passive tags, which was done considering distance from the reader which ranged from 0 feet to 25 feet, and considered the orientations of the tag, which are $0^{\circ}, 45^{\circ}, 90^{\circ}$, and $180^{\circ}$, and the common

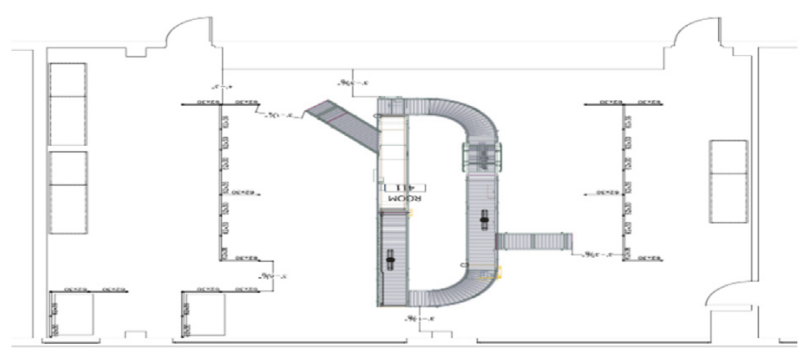

FIGURE 1: CAD drawing of the Lab where the tests were performed.

medium used, which is air, cardboard, and metal. One of the main reason for selecting these parameters is based on the industry applications. Though there were several protocols and methods developed in the past, our idea is to present a standardized test procedure to test the tags for readability and reliability based on distance from reader, medium, and orientation. There are several motivating factors which are significant to this research. The real time industry application comprises tracking inventory which usually occurs at a shorter distance. For this reason we will test tags between 0 and 25 feet. The main reason for trying on cardboard, air, and metal is for its varied application; a lot of bulk goods (apparel, books, etc.) are usually packed in cardboard. We wanted to check how would the response of tag change based on the medium it is placed. Orientation was taken into consideration because not all tracking goods are exactly at the line of sight at $0^{\circ}$ or straight facing to the handheld reader or antenna. For our method to be valuable, functional, and effective, several qualities are required which are flexibility, universality consistency, and so on [14] which have been incorporated in out method.

\section{Methodology}

The RAID labs follow a specific methodology for the testing of the tags. This method follows a Design for Six Sigma-Research methodology. Our statistical, experimental design comes from the standard best practices of engineering statistics. These methods serve as the baseline for all procedures in the RAID lab. Every experiment has its own parameters, but must follow the previously mentioned rules and regulations. The CAD drawing of the RAID lab is presented in Figure 1; the tests were performed in this lab. These tests are repeatable under normal humidity conditions and under the given system specifications.

\subsection{Assumptions}

(1) The tests are repeatable in natural under normal humidity conditions (35\%-45\%) and under the specified system configuration.

(2) The tests were performed in the lab with minimum extraneous influence in the lab.

3.2. Apparatus. The design of experiment dictates that a certain procedure must be followed, but to follow that procedure 


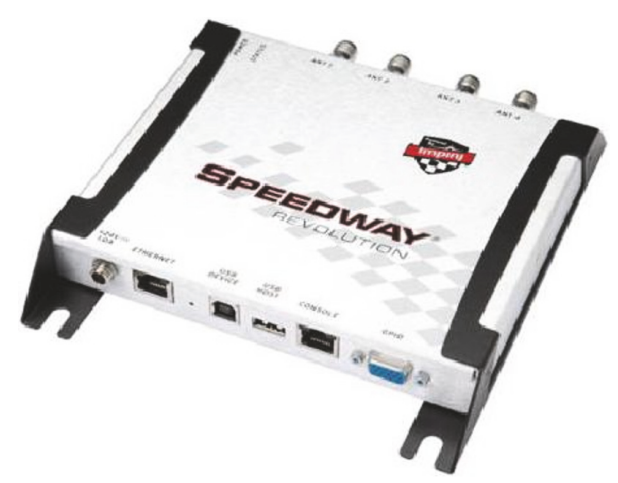

FIGURE 2: Impinj Speedway Fixed Reader.

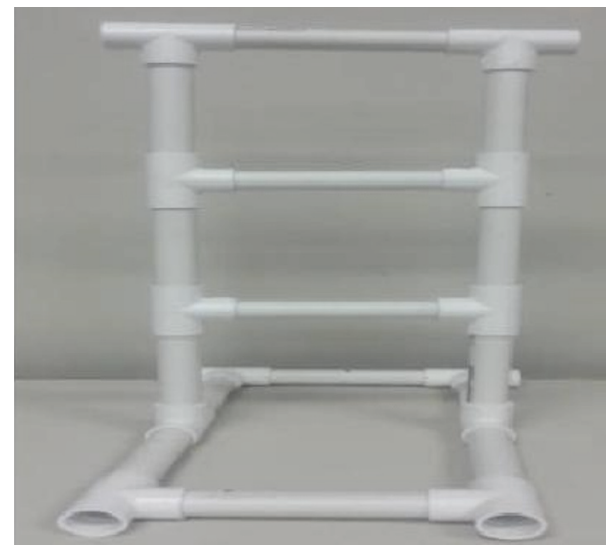

FIGURE 3: PVC Stand for holding tags.

we need a specific set of equipment. The equipment used will be the same that is dictated in the RAID lab guidelines.

(1) One Dell Inspiron 15 Laptop Computer with Impinj Speedway R-420 (Figure 2), 4 Port-FCC.

(2) UHF Fixed RFID reader.

(3) One-Far Field LHP Antenna-FCC.

(4) $2 \mathrm{ft}$ Tall PVC Stand Construct for holding the tags showed in (Figure 3).

(5) Adjustable Stand for holding the Antenna.

(6) One-Tape Measure for measuring the max distances.

(7) 6 " by 6 " by .125 .

(8) One-16 Gauge steel Plate.

(9) One- 6 " by 6 " by $.125^{\prime \prime}$ Honeycomb.

(10) Cardboard Plate.

(11) 100 Multisurface RFID tags. The tags used were RAID ID's 501, 502, 503, 504, 505, 506, 507, 508, 509, and 510.

In addition to these tools, we utilized the facilities at UT Arlington and several data analysis (Excel, MATLAB) software. These tools were all examined before, during, and after the testing to make sure that they remained in working

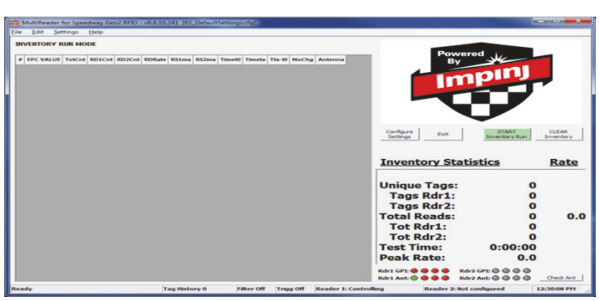

FIGURE 4: The interface of Impinj software.

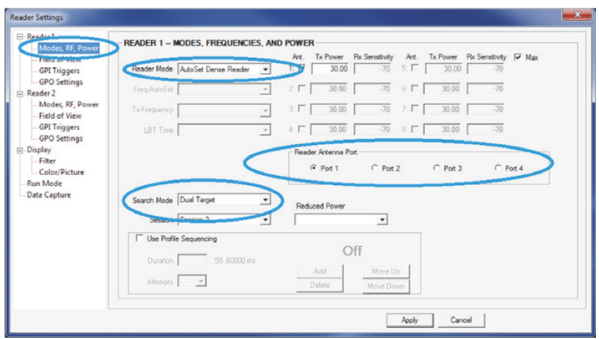

Figure 5: Reader setting.

condition. In this experiment four parameters are considered: tag quantity, orientation, distance, and material. 10 different tags are taken into consideration. The tags are tested on three mediums; air, metal, and cardboard. The third dimension of this study is distance; the 6 distances are $5 \mathrm{ft}, 10 \mathrm{ft}, 15 \mathrm{ft}, 20 \mathrm{ft}$, $25 \mathrm{ft}$, and maximum distance. The fourth and final dimension is orientation. Four orientations of 0, 45, 90, and 180 degrees are used. A complex data taking method is ensured. Each tag must be tested on each level of each parameter.

3.3. Setup for Testing. The procedure for testing of a tag is as follows. The tags, except for the one that is to be tested, are placed in a secure location where they cannot be read or interfere with the readings. The Impinj Speedway R420 fixed reader is attached to the Dell Inspiron 15 laptop. The Far Field LHP Antenna is then attached to the reader and the laptop computer is then turned on, and the Impinj MultiReader 6.6.10.240 for Speedway Gen 2 RFID software is opened. The system configuration is set as shown in Figures 4 and 5. The 10 -second scan option is selected. The testing is now ready to begin.

The test time (Figure 4) is taken as 10 seconds to ensure good readability. The reader mode (Figure 5) is selected as the Auto set Dense reader mainly because the reader automatically senses the environment and adjusts the mode accordingly. The default value of Tx power (Transmit power) $30 \mathrm{dBm}$ is used for testing purpose and the RX Sensitivity is taken as max which ensures that all the tags are read irrespective of the signal strength.

3.4. Tag Testing. For tag testing, 10 samples of each tag type were taken and evaluated for different orientations (4), different medium (3), and different distances (6), which constitutes 7200 readings. A tag is taken and is tested for readability at different distances and different orientations 


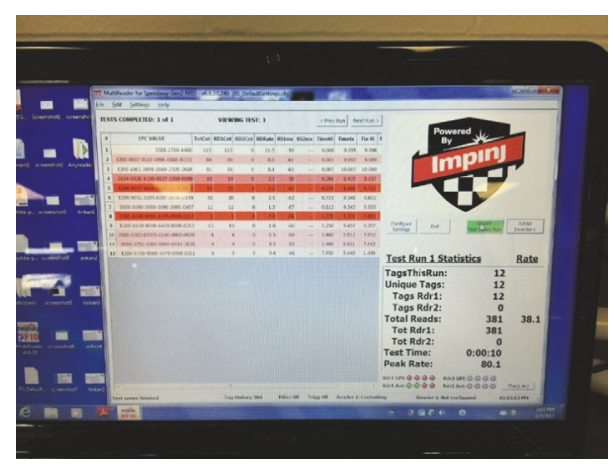

FIGURE 6: RSSI values are shown for read time of 10 seconds.

and on different mediums which are air, cardboard, and metal sheet. The tag that is to be tested is placed on the PVC Stand Construct, between the two vertical pipes, and between the two horizontal sections at the top. The tag is initially placed at 0 degrees, in air, and at 5 feet. Then all personnel are moved behind the antenna so that there is no chance for interference. The scan button on the Impinj software is pressed, and the tag is read for a period of 10 seconds. The team then changes the orientation to 45 degrees, and the procedure is repeated. The orientation is then changed again, and this is one until all orientations have been tried. The Impinj software provides the Read Signal Strength Intensity (RSSI) value. The values are then recorded for analysis. Once the RSSI values (Figure 6) have been recorded for each orientation the PVC Stand Construct is moved to $10 \mathrm{feet}$, and the whole process is repeated. The PVC Stand is subsequently moved to the remaining distances following the same procedure for the duration of that tag. The tag is then put back into the secure area.

3.5. Testing the Same Tag Type. Once a tag of a specific type is finished, the computer and fixed reader are both switched off. The operator counts to ten and then turns them both back on. This is to ensure that the data being taken is not skewed by a random good run of the equipment. Then a second tag of the same type is selected from the secure area. Then the tag Setup and tag Testing Sections are repeated.

3.6. Changing the Tag Types. After ten tags of a single make and model have been tested, the operator and research staff begin a new type of tag. The procedures are then repeated until all ten types of tags are finished in all the orientations and mediums. At this point in the process all the data for all ten types of tags has been recorded to the RAID lab data base, and the data analysis can begin. Several sets of researchers operated the reader and antenna and took data. This is to ensure that we have as little inherent human error as possible in our data set. The methodology of the RAID Labs is based on consistency and repeatability. Our goal is to provide an unbiased account of the data gathered and to bring you impartial analysis. Table 1 gives the RAID ID for the ten tags used; we considered 4 companies which manufacture multisurface passive tags.
TABLE 1: Tag ID.

\begin{tabular}{lc}
\hline Tag maker & RAID ID \\
\hline Company 1 & 501 \\
Company 1 & 502 \\
Company 1 & 503 \\
Company 2 & 504 \\
Company 2 & 505 \\
Company 3 & 506 \\
Company 3 & 507 \\
Company 3 & 508 \\
Company 4 & 509 \\
Company 4 & 510 \\
\hline
\end{tabular}

\section{Results and Discussions}

The results section of this report constitutes most of the paper. Due to the large quantity of information the section is broken up into 3 stages: summary, analysis of benchmarking, and indepth tag analysis. The data, and additional graphs, will be in the appendices. The summary stage will give a brief analysis of the other two sections. It will talk about how your tag is placed in the study and give a brief analysis specific to your tags. The analysis of benchmarking stage will be entirely devoted to providing a holistic review of all the tag performances. It will contain data on how all the tags performed over the specified parameters: distance, orientation, and material. The final stage will be in-depth tag analysis. This stage will give you details about your specific tag. It will talk about your strengths and weaknesses and provide more details as to why you are placed the way you did.

4.1. Summary. The summary section of the results section is meant to provide a quick overall analysis that will list how each tag performed over the three variable parameters: Distance, orientation, and material. This section will focus on 4 main graphs: Readability by distance, readability by orientation, readability by material, and The RAID quadrant. The three graphs of readability are summary graphs, in which each tag's readability is shown by the sublevels of each of the given parameters. The RAID quadrant will showcase the overall readability of the tag by the precision of the tag readings. Read rate percentage is defined as the number of tags in percentage read at the antenna's field of view, which acts as the system's performance indicator when comparing RFID infrastructure [12]. The readability, or read rate, of a tag is measured by taking the theoretical max RSSI and comparing it to the actual RSSI value of the transmission of the tag. This number is then calculated as a percentage

The two main portions of the Magic Quadrant are the precision percentage and the readability. Table 2 gives the precision percentages for all the tags in this experiment. The readability of each of the tags is determined by the RSSI value and is shown in Table 3.

4.1.1. Magic Quadrant. The RAID quadrant is the staple identifier of any tag testing procedure. The quadrant provides 
TABLE 2: Tag ranking by precision percentage.

\begin{tabular}{lcc}
\hline RAID ID & Precision $\%$ & Rank \\
\hline 501 & $31 \%$ & 8 \\
502 & $35 \%$ & 7 \\
503 & $74 \%$ & 1 \\
504 & $43 \%$ & 6 \\
505 & $55 \%$ & 3 \\
506 & $54 \%$ & 4 \\
507 & $30 \%$ & 9 \\
508 & $53 \%$ & 5 \\
509 & $0 \%$ & 10 \\
510 & $73 \%$ & 2 \\
\hline
\end{tabular}

TABLE 3: Tag ranking by readability.

\begin{tabular}{lcc}
\hline RAID ID & Readability & Rank \\
\hline 501 & $60 \%$ & 4 \\
502 & $40 \%$ & 7 \\
503 & $85 \%$ & 2 \\
504 & $58 \%$ & 5 \\
505 & $33 \%$ & 8 \\
506 & $21 \%$ & 9 \\
507 & $43 \%$ & 6 \\
508 & $76 \%$ & 3 \\
509 & $0 \%$ & 10 \\
510 & $86 \%$ & 1 \\
\hline
\end{tabular}

the tags, by RAID ID, ranked in two categories: Overall Readability and Precision. The RAID quadrant ranks tags on a scale of $0 \%$ to $100 \%$. The readability of a tag is the average of all the readings taken for that type of tag. This means that all 720 readings, 10 tags, 4 Orientations, 6 Distances, and 3 materials, are considered when providing the readability. The RAID quadrant is shown in Figure 7.

The numbers listed in the quadrant are the RAID ID numbers of the tags, found in Table 1 , the tag Index. The best place to be in the quadrant is the top right corner. This is because the precision and readability are highest in that corner. When Readability and Precision are high then the tag will read well in many different environments, orientations, and distances. When the precision is high it means that the readings are consistent and repeatable, so we know there is a high degree of consistency in the tags. As we can see there are three tags which beat the competition: 508, 510, and 503. Tag 510 has slight better readability, and tag 503 has slightly better precision, and 508 is a clear third. These tags are very good tags. Tag 509 failed to read at any distance greater than 2 feet and thus has zero for both Readability and precision. The other tags to note are 501 and 504.501 places 4 for readability, but its precision is much lower. Tag 504 has slightly greater precision than 501 but is not as good for readability.

4.1.2. Read Rate Scatterplot by Orientation. The purpose of this "orientation Scatterplot," shown in Figure 8, is to give a

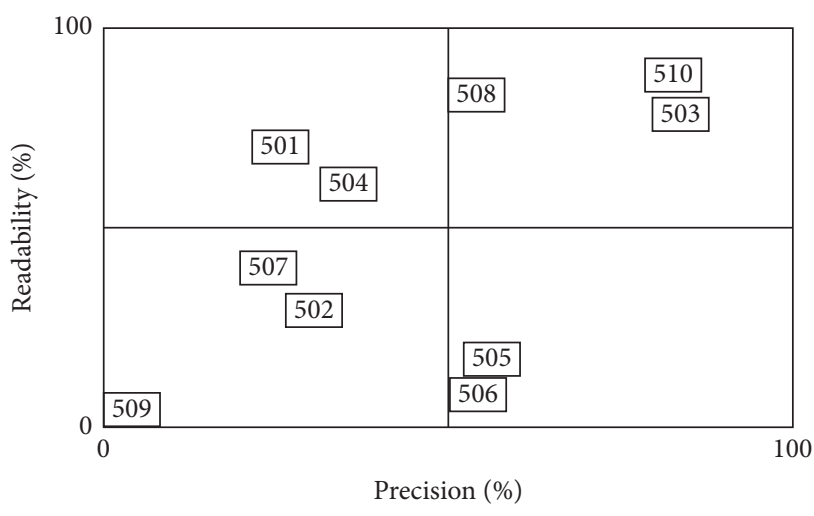

FIgURE 7: RAID performance quadrant.

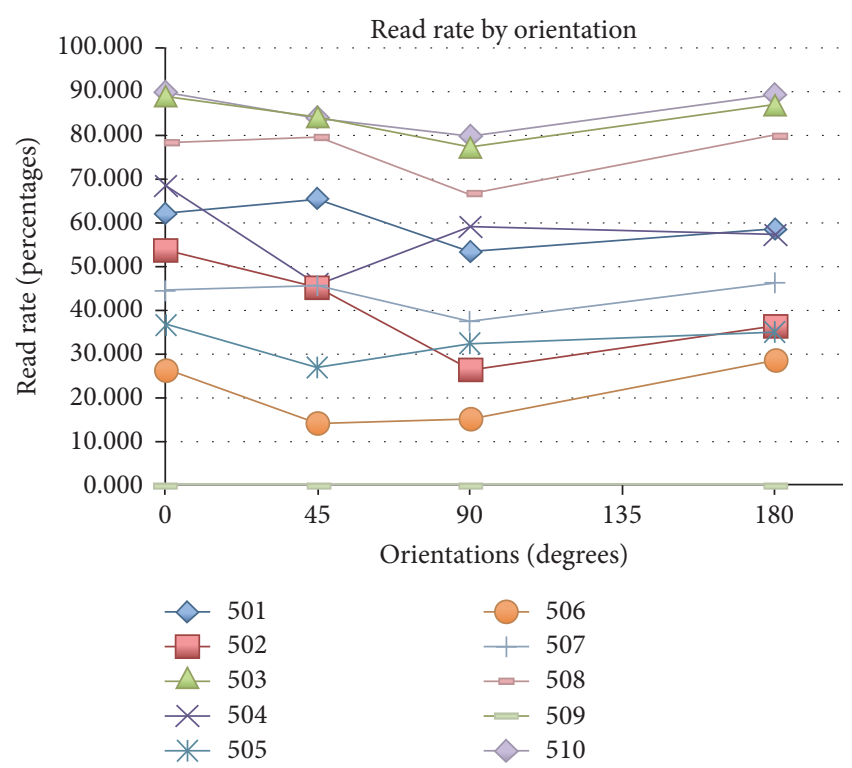

FIGURE 8: Orientation scatter plot.

general overview of individual and comparative tag performance, in terms of "Read Rate Percentages," at 4 different orientations. To test the overall competency of each of the 10 different makes of tags, orientation was one imperative factor that had to be taken into consideration.

What effect would a change in orientation of a tag have on its readability? For example, if the tag was placed on an object and that object was scanned on its side, would that make a significant difference of the tag's readability? To test this, 10 tags of each make were tested at each of the 4 orientations 15 separate times, and then averages were calculated. The graph's data points represent each make of tag's average reading percentages at each of the 4 orientations.

Figure 4 depicts two key things: first, on average, how well each tag performed at the four tested orientations $(0,45$, 90 , and 180 degrees) and second, how well the tags generally compare to each other at these orientations. The trends between each data point of each tag show whether the average reading percentages increased or decreased when orientation 


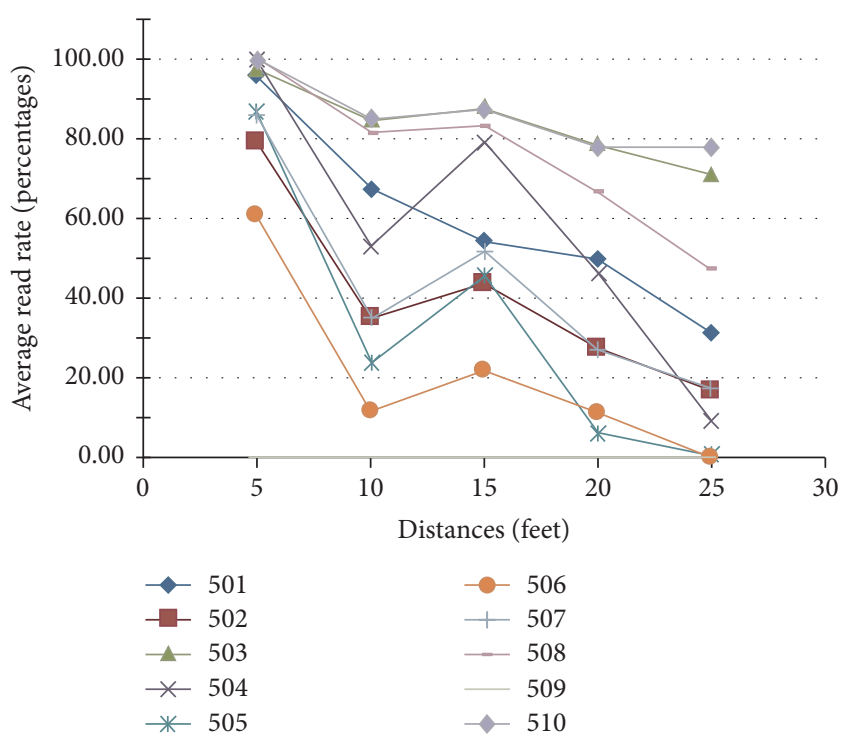

FIGURE 9: Read rate scatterplot by distance.

was changed. Different comparisons and interpretations of data can be easily read and understood. For example, per the graph, tag 510 seemed to perform best at every orientation out of all the other tags. Or maybe one would see that reading percentages for every tag, except the 502 and 504, were nearly the same within a $10 \%$ range at the orientation of 0 and 180 degrees.

4.1.3. Read Rate Scatterplot by Distance. The purpose of this "Distances Scatterplot" is to also give a general overview of individual and comparative tag performance, in terms of "reading percentages," at 5 different distances from the reader. Distance of a tag from an antenna is another imperative factor that had to be taken into consideration when discussing tag competency. How far could a tag be read? Is the readability affected by an increase (or decrease) in distance from the RFID antenna? To test this, 10 tags of each make were each tested 12 separate times at each of the 5 chosen interval distances, and then averages were calculated. The graph's data points represent each make of tag's average reading percentages at each of the 5 distances. This graph depicts two key things: first, on average, how well each tag performed at the five tested distances $(5,10,15,20$, and 25 feet) and second, how well the tags generally compare to each other at these distances. The trends between each data point of each tag show whether the average reading percentages increased or decreased when the distance was altered. Different comparisons and interpretations of data can be easily read and understood. For example, per the graph, five out of the ten types of tags read above $90 \%$ at five feet. Also, it could be noticed that, for each tag, five feet was the distance that tested to have the highest average reading percentage. This graph is shown in Figure 9.

4.1.4. Read Rate by Material. The third summary graph, Figure 10 , is a representation of the \% read of 10 different tags

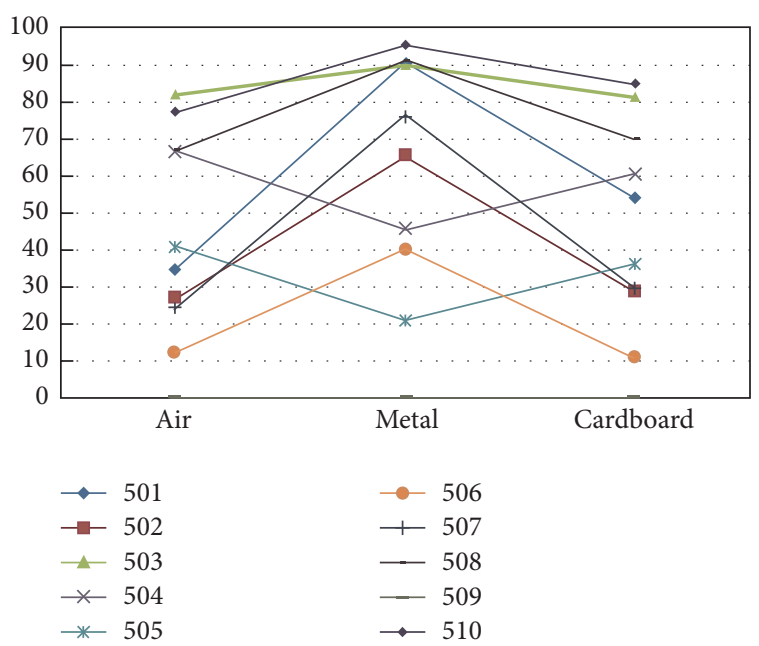

FIGURE 10: Read rate scatterplot by material.

over the 3 main surfaces: air, metal, and cardboard. The values in Figure 10 are an average of reading percentages at each of the 5 tested distances and 4 orientations. We see that majority of the tags have a higher average read rate percentage when read on a metal background. It is also clear that 509 tag were the exception to this trend. The 509 , as previously stated, was not able to read above 2 feet.

Figures 7-10 provide an overview of all the tag performances taken in this benchmarking study. The important things to note from this section are that tags have different strengths depending on the material, distance, and orientation. There does seem to be an overall trend that multisurface tags test better on metal than on any other substance. The two notable exceptions to this rule are the silver line tags (504). There is also a general decrease in the read rates as distance increases. There is also an exception to this rule. At 10 feet, tag read rates seem to decrease. This is because the Gen 2 protocol has some blind spots in its wave propagation. This is a known issue and effects all Gen 2 tags, so it should not be considered significant to this study. The final summary point is that, across the board, tags read best at 0 degrees and then at 180 degrees. Tilting the tag at all can infer some technical difficulties.

4.1.5. Analysis of Benchmarking. Summary gave a general idea of what to expect when looking at the read rates of Multisurface RFID tags. We saw the basic trends of the tags and examined an RFID phenomenon. The next step in the analysis is to look a little bit deeper into the benchmarking of these tags.

4.1.6. Decreasing Read Rates over Distance. One of the main factors that we saw in decreasing read rates was that, as distance increases, readability decreases. This means that when we go out to 25 feet, not many of the tags read well. In fact, in Table 4, we see the difference, in read rates of tags at $5 \mathrm{ft}$ and $25 \mathrm{ft}$ when placed on metal.

In Table 4, we see that the same tags are placed in the top 5, but not necessarily in the same order. This is because the tags 
TABLE 4: Read rates of tags on metal at $5 \mathrm{ft}$ and $25 \mathrm{ft}$.

\begin{tabular}{lccccc}
\hline \multicolumn{5}{c}{} & \multicolumn{3}{c}{ Metal } & $\begin{array}{c}25 \mathrm{ft} . \\
\text { Tft. }\end{array}$ & \multicolumn{3}{c}{} \\
Rank & Tag type & Avg & Rank & Tag type & Avg \\
\hline 1 & 508 & 111.75 & 1 & 510 & 86.15 \\
2 & 510 & 107.1 & 2 & 501 & 81.35 \\
3 & 503 & 103 & 3 & 503 & 78.8 \\
4 & 507 & 102.1 & 4 & 508 & 73.25 \\
5 & 501 & 101.15 & 5 & 507 & 50.5 \\
6 & 502 & 101.15 & 6 & 502 & 47.3 \\
7 & 504 & 95.4 & 7 & 504 & 2.15 \\
8 & 505 & 81.1 & 8 & 505 & 0 \\
9 & 506 & 80.05 & 9 & 506 & 0 \\
10 & 509 & 0 & 10 & 509 & 0 \\
\hline
\end{tabular}

TABLE 5: Average max distance by per tag.

\begin{tabular}{lccc}
\hline \multirow{2}{*}{ RAID ID } & \multicolumn{2}{c}{ Average max FT } & \multirow{2}{*}{ Cardboard } \\
& Air & Metal & \\
\hline 501 & $23 \mathrm{ft}$ & $52 \mathrm{ft}$ & $22 \mathrm{ft}$ \\
502 & $21 \mathrm{ft}$ & $27 \mathrm{ft}$ & $20 \mathrm{ft}$ \\
$\mathbf{5 0 3}$ & $\mathbf{4 1} \mathrm{ft}$ & $\mathbf{6 2} \mathrm{ft}$ & $\mathbf{3 8 f t}$ \\
504 & $28 \mathrm{ft}$ & $32 \mathrm{ft}$ & $32 \mathrm{ft}$ \\
505 & $7 \mathrm{ft}$ & $9 \mathrm{ft}$ & $7 \mathrm{ft}$ \\
506 & $15 \mathrm{ft}$ & $18 \mathrm{ft}$ & $6 \mathrm{ft}$ \\
507 & $13 \mathrm{ft}$ & $26 \mathrm{ft}$ & $13 \mathrm{ft}$ \\
508 & $28 \mathrm{ft}$ & $30 \mathrm{ft}$ & $25 \mathrm{ft}$ \\
509 & $1 \mathrm{ft}$ & $2 \mathrm{ft}$ & $1 \mathrm{ft}$ \\
$\mathbf{5 1 0}$ & $\mathbf{3 8 f t}$ & $\mathbf{6 8 f t}$ & $\mathbf{4 5 f t}$ \\
\hline
\end{tabular}

attenuate over distance, and some tags attenuate faster than others. Another important note to make is that some tags scored over $100 \%$ on average for their read rate; how is this possible? Since the read rate is a function of the theoretical max intensity of the RF wave returning to the reader, it is possible that the wave is stronger. This increased strength is most likely due to natural ambient RF waves. One of the reasons could be due to the reflection of ways in an enclosed room (lab) where the experiment was conducted. The final thing to point out on this graph is that several tags did not read on metal at $25 \mathrm{ft}$, and another tag barely read at all. Tags 505, 506, and 509 do not appear to be good tags for longer range RFID systems. Table 5 shows the average max distances to the nearest foot. The tags in the table do not necessarily hold the same rank as of those in the readability charts because the readability charts only consider data in the first $25 \mathrm{ft}$, and this chart considers data that goes up to almost 70 feet.

Judging by the data that is in the table, we can see that the tag that is best for use in distance is RAID Tag 510, and the second best is RAID Tag 503. They are again both very close, and we can see they are much better than the next in this class. 501 also shows a lot of promise but does not quite make the $25 \mathrm{ft}$ mark on all its three categories.
TABLE 6: Correlation coefficients.

\begin{tabular}{lc}
\hline To/from & $5 \mathrm{ft}$ \\
\hline $5 \mathrm{ft}$ & 1 \\
$10 \mathrm{ft}$ & 0.727135 \\
$15 \mathrm{ft}$ & 0.857618 \\
$20 \mathrm{ft}$ & 0.765536 \\
$25 \mathrm{ft}$ & 0.619915 \\
\hline
\end{tabular}

TABLE 7: Slope of the trend over distance by tag.

\begin{tabular}{lc}
\hline Tags & Slopes \\
\hline 501 & -2.93 \\
502 & -2.65 \\
503 & -1.19 \\
504 & -3.75 \\
505 & -3.79 \\
506 & -2.44 \\
507 & -2.91 \\
508 & -2.47 \\
510 & -1.01 \\
\hline
\end{tabular}

To have a better idea of what kind of depreciation we see over distance, we need to examine two separate things: Correlation Analysis and the slope of the line over distance. The first of these two things will show us how correlated to distance the depreciation in read rate is.

The numbers in the Table 6 represent the correlation coefficients of the depreciation in read rates over distance. These numbers represent how dependent the other distances are on the value of the read rate at $5 \mathrm{ft}$. The expectation for this chart is that the bigger the distance is, the less correlated the values will be, but as we saw earlier, there is an anomaly at 10 feet. Other than that, the graph appears to be exponentially decaying.

Table 7 shows the slope of the line drawn between the read rates of tags placed at $5,10,15,20$, and 25 feet. This chart is important because it tells us how quickly the tags lose their strength, or attenuate.

Table 7 shows the slope of the line, giving the trend of how tags act over distance. The number in each box gives the decrease in the read rate for each foot on average from 5 feet to 25 feet. This allows us to predict at any given point the theoretical value of read rate for any given tag. This is an average for all orientations and all materials. That means that there will be some error in our estimations, but it is a good place to start from.

The tables and figures above give us an accurate picture as to how any given tags will act at any given time over distance.

4.1.7. In-Depth Tag Analysis. See Tables 8, 9, and 10. In this section, we presented the in-depth analysis of three tags only to showcase how we performed the analysis. The data is broken down first by the tag tested, listed under the tag type. It is then broken down by the material it is placed on, then by the orientation angle of the tag about the reader, and finally 
TABLE 8: Tag 501.

\begin{tabular}{|c|c|c|c|c|c|c|c|c|c|c|c|c|c|}
\hline \multirow{3}{*}{$\begin{array}{l}\text { Tag type } \\
\text { Material } \\
\text { Orientation }\end{array}$} & \multicolumn{12}{|c|}{ Tag 501} & \multirow{3}{*}{ Cumu. \% } \\
\hline & \multicolumn{4}{|c|}{ Air } & \multicolumn{4}{|c|}{ Metal } & \multicolumn{4}{|c|}{ Cardboard } & \\
\hline & 0 & 45 & 90 & 180 & 0 & 45 & 90 & 180 & 0 & 45 & 90 & 180 & \\
\hline 5 feet & $100 \%$ & $100 \%$ & $100 \%$ & $100 \%$ & $100 \%$ & $100 \%$ & $100 \%$ & $100 \%$ & $100 \%$ & $100 \%$ & $100 \%$ & $100 \%$ & $100 \%$ \\
\hline 10 feet & $70 \%$ & $100 \%$ & $100 \%$ & $10 \%$ & $100 \%$ & $100 \%$ & $100 \%$ & $100 \%$ & $60 \%$ & $100 \%$ & $100 \%$ & $30 \%$ & $81 \%$ \\
\hline 15 feet & $0 \%$ & $0 \%$ & $0 \%$ & $50 \%$ & $100 \%$ & $100 \%$ & $100 \%$ & $100 \%$ & $70 \%$ & $90 \%$ & $20 \%$ & $100 \%$ & $61 \%$ \\
\hline 20 feet & $30 \%$ & $0 \%$ & $0 \%$ & $30 \%$ & $100 \%$ & $100 \%$ & $100 \%$ & $100 \%$ & $90 \%$ & $70 \%$ & $40 \%$ & $70 \%$ & $61 \%$ \\
\hline 25 feet & $0 \%$ & $0 \%$ & $0 \%$ & $10 \%$ & $100 \%$ & $100 \%$ & $90 \%$ & $100 \%$ & $30 \%$ & $10 \%$ & $0 \%$ & $20 \%$ & $38 \%$ \\
\hline Cumu. \% & $40 \%$ & $40 \%$ & $40 \%$ & $40 \%$ & $100 \%$ & $100 \%$ & $\mathbf{9 8} \%$ & $100 \%$ & $70 \%$ & $74 \%$ & $52 \%$ & $64 \%$ & \\
\hline
\end{tabular}

TABle 9: Tag 503.

\begin{tabular}{|c|c|c|c|c|c|c|c|c|c|c|c|c|c|}
\hline \multirow{3}{*}{$\begin{array}{l}\text { Tag type } \\
\text { Material } \\
\text { Orientation }\end{array}$} & \multicolumn{12}{|c|}{ Tag 503} & \multirow{3}{*}{ Cumu. \% } \\
\hline & \multicolumn{4}{|c|}{ Air } & \multicolumn{4}{|c|}{ Metal } & \multicolumn{4}{|c|}{ Cardboard } & \\
\hline & 0 & 45 & 90 & 180 & 0 & 45 & 90 & 180 & 0 & 45 & 90 & 180 & \\
\hline 5 feet & $100 \%$ & $100 \%$ & $80 \%$ & $80 \%$ & $100 \%$ & $100 \%$ & $100 \%$ & $100 \%$ & $100 \%$ & $100 \%$ & $80 \%$ & $100 \%$ & $\mathbf{9 5} \%$ \\
\hline 10 feet & $70 \%$ & $30 \%$ & $30 \%$ & $0 \%$ & $90 \%$ & $60 \%$ & $90 \%$ & $60 \%$ & $50 \%$ & $40 \%$ & $40 \%$ & $10 \%$ & $48 \%$ \\
\hline 15 feet & $80 \%$ & $60 \%$ & $10 \%$ & $50 \%$ & $100 \%$ & $100 \%$ & $40 \%$ & $90 \%$ & $70 \%$ & $70 \%$ & $0 \%$ & $30 \%$ & $\mathbf{5 8} \%$ \\
\hline 20 feet & $30 \%$ & $10 \%$ & $10 \%$ & $0 \%$ & $100 \%$ & $90 \%$ & $30 \%$ & $100 \%$ & $50 \%$ & $30 \%$ & $0 \%$ & $0 \%$ & $38 \%$ \\
\hline 25 feet & $0 \%$ & $0 \%$ & $0 \%$ & $0 \%$ & $100 \%$ & $70 \%$ & $10 \%$ & $80 \%$ & $0 \%$ & $0 \%$ & $0 \%$ & $20 \%$ & $23 \%$ \\
\hline Cumu. \% & $56 \%$ & $40 \%$ & $26 \%$ & $26 \%$ & $\mathbf{9 8} \%$ & $84 \%$ & $54 \%$ & $86 \%$ & $54 \%$ & $48 \%$ & $24 \%$ & $32 \%$ & \\
\hline
\end{tabular}

TABLE 10: Tag 502.

\begin{tabular}{|c|c|c|c|c|c|c|c|c|c|c|c|c|c|}
\hline \multirow{3}{*}{$\begin{array}{l}\text { Tag type } \\
\text { Material } \\
\text { Orientation }\end{array}$} & \multicolumn{12}{|c|}{ Tag 502} & \multirow{3}{*}{ Cumu. \% } \\
\hline & \multicolumn{4}{|c|}{ Air } & \multicolumn{4}{|c|}{ Metal } & \multicolumn{4}{|c|}{ Cardboard } & \\
\hline & 0 & 45 & 90 & 180 & 0 & 45 & 90 & 180 & 0 & 45 & 90 & 180 & \\
\hline 5 feet & $100 \%$ & $100 \%$ & $100 \%$ & $100 \%$ & $100 \%$ & $100 \%$ & $100 \%$ & $100 \%$ & $100 \%$ & $100 \%$ & $100 \%$ & $100 \%$ & $100 \%$ \\
\hline 10 feet & $100 \%$ & $100 \%$ & $100 \%$ & $100 \%$ & $100 \%$ & $90 \%$ & $100 \%$ & $100 \%$ & $100 \%$ & $90 \%$ & $100 \%$ & $100 \%$ & $\mathbf{9 8} \%$ \\
\hline 15 feet & $100 \%$ & $100 \%$ & $100 \%$ & $100 \%$ & $100 \%$ & $100 \%$ & $100 \%$ & $100 \%$ & $100 \%$ & $100 \%$ & $100 \%$ & $100 \%$ & $100 \%$ \\
\hline 20 feet & $100 \%$ & $100 \%$ & $90 \%$ & $100 \%$ & $100 \%$ & $100 \%$ & $90 \%$ & $100 \%$ & $100 \%$ & $100 \%$ & $80 \%$ & $100 \%$ & $\mathbf{9 7} \%$ \\
\hline 25 feet & $90 \%$ & $60 \%$ & $90 \%$ & $100 \%$ & $100 \%$ & $100 \%$ & $70 \%$ & $100 \%$ & $100 \%$ & $100 \%$ & $90 \%$ & $100 \%$ & $\mathbf{9 2} \%$ \\
\hline Cumu. \% & $98 \%$ & $92 \%$ & $96 \%$ & $100 \%$ & $100 \%$ & $\mathbf{9 8} \%$ & $92 \%$ & $100 \%$ & $100 \%$ & $98 \%$ & $\mathbf{9 4} \%$ & $100 \%$ & \\
\hline
\end{tabular}

by the distance, which can be found on the leftmost column of each table.

In each cell of the table there is a percentage read, and that is the percent of the trials in which a single tag was placed in view and identified. The total at the bottom of each column is the percent chance that a tag will read a random distance equal to or less than 25 feet. The cumulative total column on the right shows the percent chance that a tag will read on a random material. Some tags read better than others, and there are some patterns in the data that are important to point out.

Table 8 shows the data summary for the tag 501. The tag read $100 \%$ of the time at 5 feet, regardless of orientation and distance. However, when the tags were placed at $25 \mathrm{ft}$ the read percentage dropped to $38 \%$. The tags also read $98 \%$ of the time on metal regardless of orientation. It read better on cardboard than on air, and the only time it did not read at all on cardboard is at 25 feet at an orientation of 90 degrees to the antenna. The tags were hardest to read at 90 degrees. This tag performed well overall, and its only area for improvement is at the previously mentioned 90 degree orientation.

The second tag we tested was tag 503, and its data summary can be found in Table 9. This tag did not perform as highly as the Universal Asset, but still performed well. At 5 feet it read $95 \%$ of the time, but only $23 \%$ of the time at 25 feet, across all orientations and materials. This tag also performed decently in open air, well on cardboard, and very well on metal. If the tag is placed at 0,45 , or 180 degrees and on metal it is at least $84 \%$ likely to read. This further confirms the idea that tags work best on metallic surfaces

The third and final tag is the tag 502 and it can be found in Table 10. This tag is off the charts in terms of performance. It either ties or outperforms both other tags in every category. There is no category that the tag reads at less than $92 \%$. This tag reads so well that even in open air at 90 degrees and 25 
feet it will read $90 \%$ of the time. This area has typically been the most challenging for tags to perform well in. The exact max distance for this tag has not been determined yet, but it is greater than 35 feet. This is by far the most versatile of all the investigated tags so far.

\section{Conclusion and Future Work}

When looking back over all the data and seeing all the charts and graphs together, it is hard to put together a specific ranking for all ten tags. The two top tags are easily the 503 and 510, but after that the only thing that is clear is that the 509 appears to be the worst. There are several things that could be said for the 501 and 502, but the most accurate is that they are consistently placed in the top 5 tags tested. There are several graphs that depict the strengths and weaknesses of each of the tags. No tag scored $90 \%$ or higher for either readability or precision, so there is room for growth. However, some tags seem to place well on every metric given.

The benchmarking of these tags is comprehensive, and a significant number of additional charts and graphs can be found in the appendix. It is time to address what the future steps of this process could look like. The current setup for the experiment dealt with only a tag, at one angle to the antenna, with only one antenna, and at a fixed height. This was done as a standard of tag testing protocol. However, it does raise a few questions about what would happen if any of those parameters was changed. Part of the reason that there is a drop off in read rate at 10 feet is because of an issue with the Gen 2 standard, and that could be examined further if additional antennas were brought into the setup. In addition to the extra antenna, another study might be to look at the optimal height of a tag, relative to the reader, or the best angle to place tags at to maximize the read rate. One of the possible future works would be to try testing in different system configuration using different readers and antennas.

This study also only considers the Multisurface RFID tags of a few companies. These companies are the leaders in this field, but a more comprehensive benchmark against all Gen 2 RFID tags could highlight the strengths of the 501, 502, and 503.

\section{Conflicts of Interest}

The authors declare that they have no conflicts of interest.

\section{Acknowledgments}

This project has been partially funded by Metalcraft Inc. (http://www.idplate.com/) and PWD Group (http://pwdgroups .com/).

\section{References}

[1] E. C. Jones and C. A. Chung, RFID in Logistics, 2009.

[2] E. C. Jones, B. Gray, M. Wijemanne, and J. Bolton, Tracked: What You Should Know about RFID, Big Data, The Internet of Things, and Data Security, Arlington: PWD Group Press, 2015.
[3] J. H. Ryu, M. Irfan, and A. Reyaz, "A review on sensor network issues and robotics," Journal of Sensors, vol. 2015, Article ID 140217, 2015.

[4] K. M. Ramakrishnan and D. D. Deavours, "Performance benchmarks for passive UHF RFID tags," in Proceedings of the 2006 13th GI/ITG Conference on Measuring, Modelling and Evaluation of Computer and Communication Systems, MMB 2006, deu, March 2006.

[5] P. V. Nikitin and K. V. S. Rao, "Performance of RFID tags with multiple RF ports," in Proceedings of the 2007 IEEE Antennas and Propagation Society International Symposium, AP-S, pp. 5459-5462, usa, June 2007.

[6] W. B. Zou, Y. Wu, and Y. Zhao, "Automatic testing system for UHF passive RFID tag performance," in Proceedings of the 2009 International Conference on Networking and Digital Society, ICNDS 2009, pp. 79-82, chn, May 2009.

[7] G. Marrocco, E. Di Giampaolo, and R. Aliberti, "Estimation of UHF RFID reading regions in real environments," IEEE Antennas and Propagation Magazine, vol. 51, no. 6, pp. 44-57, 2009.

[8] V. Caredda, P. F. Orrú, G. Romagnoli, A. Volpi, and F. Zedda, "RFID technology for blood tracking: An experimental approach for benchmarking different devices," International Journal of RF Technologies: Research and Applications, vol. 7, no. 4, pp. 209-228, 2016.

[9] J. D. Porter, R. E. Billo, and M. H. Mickle, "A standard test protocol for evaluation of radio frequency identification systems for supply chain applications," Journal of Manufacturing Systems, vol. 23, no. 1, pp. 46-55, 2004.

[10] L. Sirico and B. Martin, Long range RFID Benchmark Test Results, RFID Global Solution Inc., 2009.

[11] S. Piramuthu, A. Rizzi, G. Vignali, and A. Volpi, "Benchmarking of RFID devices for apparel applications: An experimental approach," International Journal of RF Technologies: Research and Applications, vol. 6, no. 2-3, pp. 151-169, 2015.

[12] E. Bottani, G. Ferretti, R. Montanari, A. Rizzi, and A. Volpi, "Performances of RFID, acousto-magnetic and radio frequency technologies for electronic article surveillance in the apparel industry in Europe: A quantitative study," International Journal of RF Technologies: Research and Applications, vol. 3, no. 2, pp. 137-158, 2012.

[13] S. C. Cheung, W. K. Chan, M. K. Penny, M. Lionel et al., A Combinatorial Methodology for RFID Benchmarking, 2006.

[14] K. Mazur, B. Ksiezopolski, and R. Nielek, "Multilevel Modeling of Distributed Denial of Service Attacks in Wireless Sensor Networks," Journal of Sensors, vol. 2016, Article ID 5017248, 2016. 


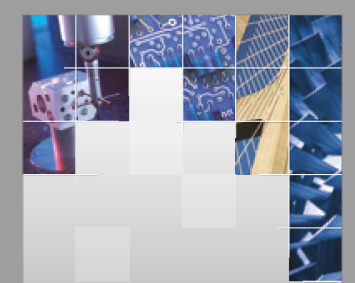

\section{Enfincering}
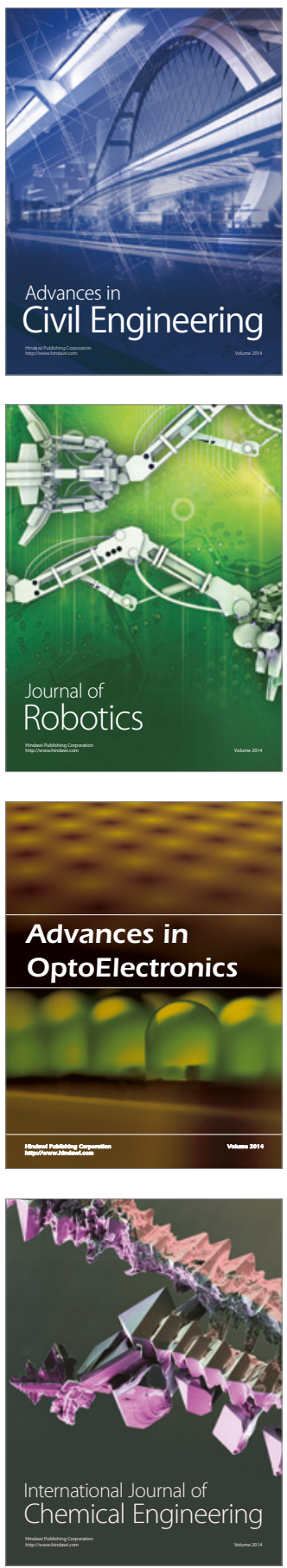

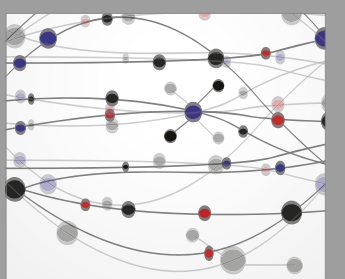

The Scientific World Journal

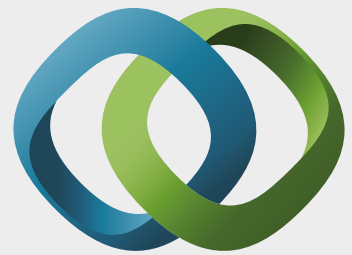

\section{Hindawi}

Submit your manuscripts at

https://www.hindawi.com
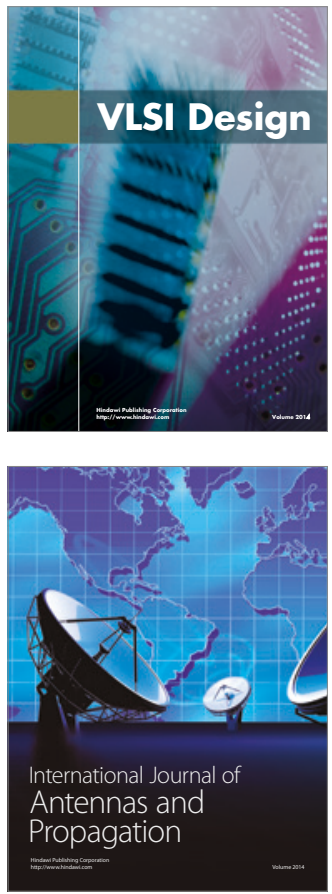

\section{Rotating}

Machinery
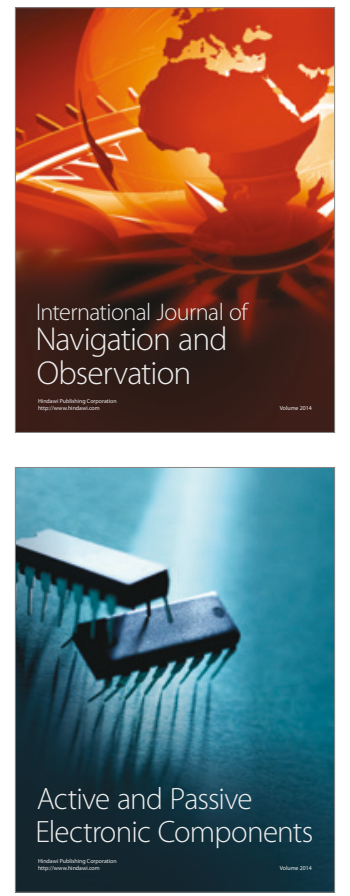
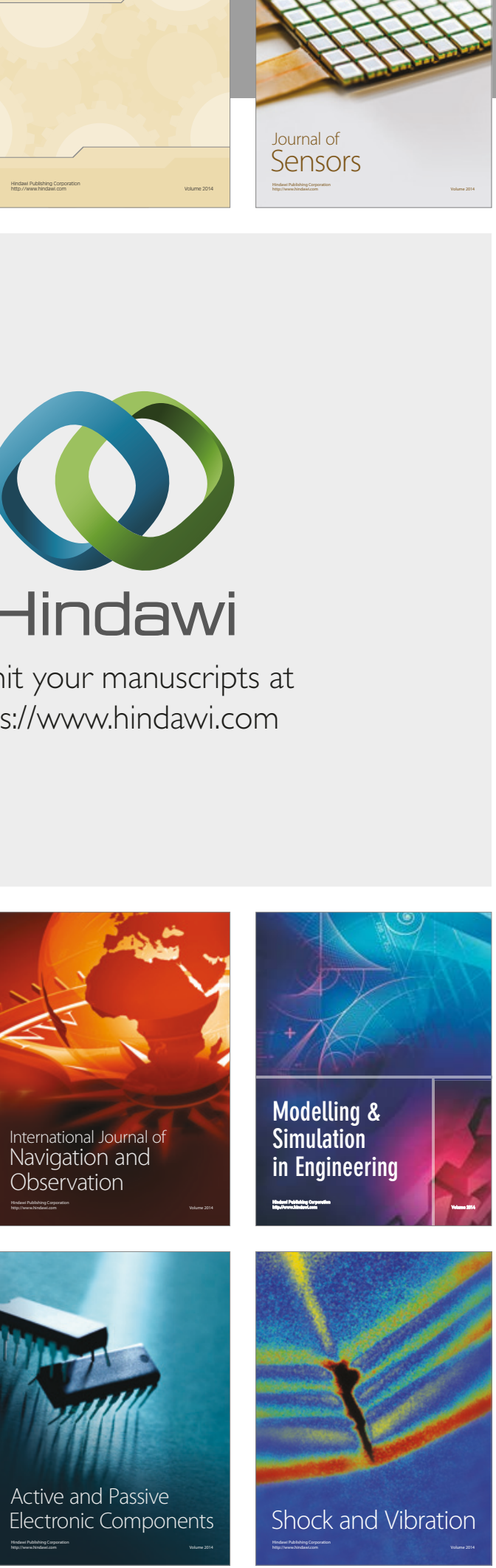
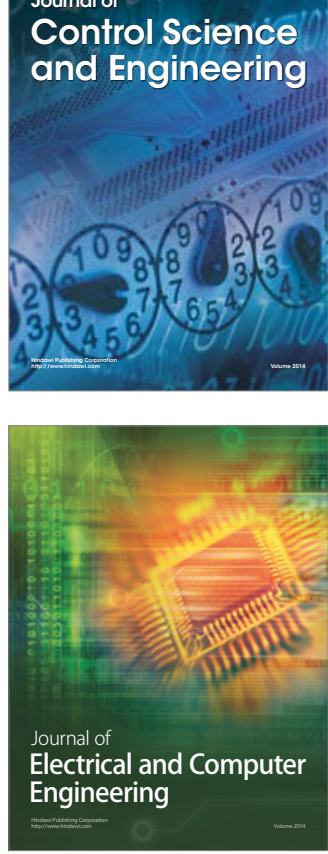

Distributed

Journal of

Control Science

and Engineering
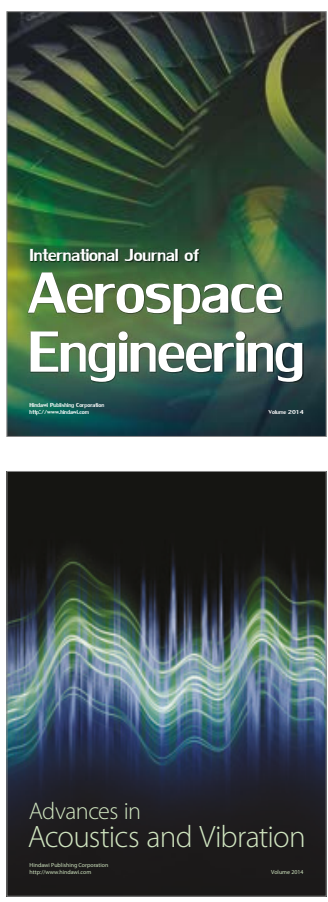

Sensor Networks 